\title{
Rapid detection in food and feed
}

\author{
Rudolf Krska • Michel Nielen
}

Published online: 23 July 2013

(C) Springer-Verlag Berlin Heidelberg 2013

Chemical contaminant monitoring in food and feed is a very relevant issue in global food quality and safety according to ongoing and previous Rapid Alert System on Food and Feed (RASFF) alerts. Also, consumers have placed chemical contaminants at the top of the "worry scale" of food-related risks. The European Food Safety Authority urgently needs reliable exposure and toxicity data for many (emerging) contaminants such as perfluorinated compounds and plant and marine toxins. According to Regulation (EC) No. $178 / 2002$, the food and feed industries are responsible for the safety of their products. In addition, Regulation (EC) No. $882 / 2004$ requires that official controls are conducted, taking into account identified risks. Therefore, the demand for simplified and rapid test methods at critical control points in the entire chain has never been greater. At the same time, such novel screening tools should have multianalyte, multiclass (multiplex) capability by detecting as many contaminant parameters in parallel as possible within the shortest period of time.

This topical collection on rapid detection in food and feed addresses this urgent demand for rapid multianalyte testing. The articles collected in this issue reflect the latest state of the art and the current and future challenges in rapid detection in food and feed. On the one hand, these articles include novel methods for the rapid quantification of food and feed contaminants by using established high-performance analytical techniques such as liquid chromatography-tandem mass spectrometry. On the other hand, major emphasis is given to innovative methods such as near-infrared hyperspectral imaging, quantum-dot-loaded liposomes for ultrasensitive onsite determination and easy-to-use multiplex dipstick assays. The methods presented have been developed and in many cases validated through interlaboratory comparison for a wide range of (emerging) contaminants in food and feed. These include mycotoxins in cereals and milk products, antibiotics in poultry muscle and in honey, brominated flame retardants in fish and inorganic arsenic in rice.

We would like to express our great gratitude to the contributors; thanks to them this issue provides updated and high-quality original contributions on new developments in rapid detection in food and feed, its emerging applications and a picture of future trends. We hope that the collection of these articles stimulates further research to develop simplified and rapid test methods for the determination of contaminants in the entire food chain as an important means to protect consumers. Last but not least, we are also very thankful to the editorial team and the reviewers of Analytical and Bioanalytical Chemistry for their support and fantastic cooperation.

Published in the topical collection Rapid Detection in Food and Feed with guest editors Rudolf Krska and Michel Nielen.

\footnotetext{
R. Krska $(\bowtie)$

Department for Agrobiotechnology (IFA-Tulln), University of Natural Resources and Life Sciences Vienna, Konrad-Lorenz-Str. 20, 3430 Tulln, Austria

e-mail: rudolf.krska@boku.ac.at

M. Nielen

RIKILT Institute of Food Safety, Wageningen UR, P.O. Box 230, 6700 AE Wageningen, The Netherlands

e-mail: michel.nielen@wur.nl
} 


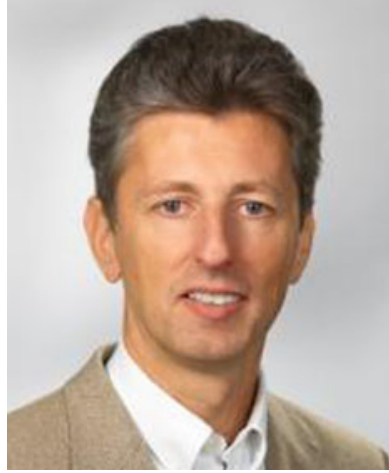

Rudolf Krska is a full Professor of (bio)analytics and organic trace analysis and is Head of the Department for Agrobiotechnology, IFA-Tulln, with more than 150 co-workers at the University of Natural Resources and Life Sciences, Vienna (BOKU). He obtained his degree in chemistry at the Vienna University of Technology and is an expert in food and feed analysis by chromatographic, mass-spectrometric and immunoanalytical techniques. In 2009/2010 he worked for 1 year as Acting Chief of Health Canada's Food Research Division in Ottawa. $\mathrm{He}$ has received six scientific awards and is the author or co-author of more than $200 \mathrm{SCI}$ publications.

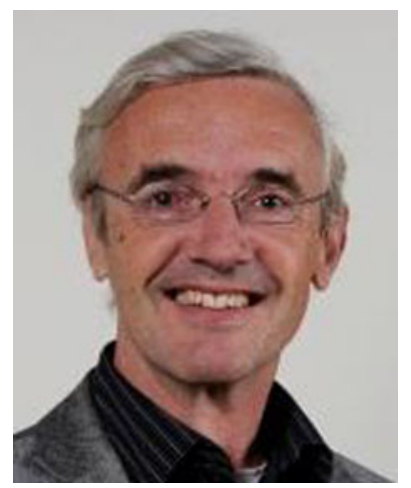

Michel Nielen is a Principal Scientist at RIKILT, Scientific Director of TI-COAST, the Dutch public-private partnership on analytical science and technology, and a Professor of Analytical Chemistry at Wageningen University. $\mathrm{He}$ obtained his degree in analytical chemistry at the Free University of Amsterdam. He is a co-founder and co-chairman of the symposium series on recent advances in food analysis. He is the author or co-author of more than 150 peerreviewed publications. 